\title{
Vida asociativa y desarrollo local en dos pueblos nicaragüenses
}

\author{
Nadia Molenaers
}

$\mathrm{L}$

as organizaciones de la sociedad civil son a menudo vistas como portadoras de capital social, y la existencia de tales organizaciones como una muestra de que se ha superado el dilema de la acción colectiva y de la disposición a confiar, intercambiar y cooperar. De esta manera, las actitudes y las estructuras serían dos elementos complementarios en el debate acerca del capital social. Este artículo postula que unas y otras no siempre van de la mano. Una investigación realizada en dos pueblos nicaragüenses demuestra que existe una gran contradicción entre la estructura de la vida asociativa y la estructura del capital social al nivel local. En vez de ser agentes de transformación social, las organizaciones más bien tienden a institucionalizar abismos locales de segregación social, política o económica. 


\title{
I \\ Rol de la sociedad civil, capital social y políticas de desarrollo
}

\begin{abstract}
Tu maíz está maduro, el mío lo estará mañana. Sería beneficioso para ambos que yo trabajara contigo hoy, y que tú me ayudaras mañana. No te tengo cariño y sé que tú tampoco lo tienes por mí. Podría entonces esforzarme, no para tu beneficio, sino para el mío propio con la expectativa de un retorno. Pero sé que seré decepcionado y que dependería en vano de tu gratitud. Entonces yo te dejo trabajar solo y tú me tratas de la misma manera. Pasan las estaciones y ambos continuamos perdiendo nuestras cosechas por falta de confianza y seguridad mutua. David Hume (1923)
\end{abstract}

La parábola de Hume ilustra un dilema fundamental de la acción colectiva: cooperar o no cooperar. Racionalmente, todos podrían estar mejor si pudiesen cooperar, pero por falta de confianza no se aprovechan los beneficios ni las oportunidades de coordinación y cooperación.

Una solución a este dilema se halla en el concepto de capital social, entendido como las redes densas y horizontales de compromiso cívico y las normas generalizadas de confianza y reciprocidad. El capital social parece ser la fuerza impulsora de la práctica democrática y de la prosperidad económica (Putnam, 1993; Harrison y Huntington, 2000; Fukuyama, 1995). En las democracias estables con altos niveles de desarrollo socioeconómico, la vida asociativa se presenta principalmente como el resultado histórico y casi natural de las experiencias de cooperación horizontal entre ciudadanos, acumuladas de abajo hacia arriba. La disposición a confiar, intercambiar y cooperar tiene estrecha relación con la existencia de ciertas estructuras, como las organizaciones y la afiliación asociativa, las que precisamente demuestran la superación del dilema de la acción colectiva. Las actitudes y las estructuras son entonces los dos elementos principales del debate acerca del capital social. Por lo tanto, la existencia de una gran cantidad de organizaciones y de altos niveles de afiliación asociativa tiende a ir mano a mano con altos niveles de confianza (Putnam, 1993 y 2000).

Los actores del desarrollo han reconocido el rol potencial en los procesos de desarrollo tanto del capital social como de una sociedad civil fuerte y vibrante $^{1}$ (Ostrom, 2001). A partir del decenio de 1990, los promotores del desarrollo internacional, como el Banco Mundial y el Programa de las Naciones Unidas para el Desarrollo, e incluso la Unión Europea y los gobiernos nacionales de occidente, claramente han elegido consolidar la sociedad civil en los países en desarrollo (PNUD, 1993, p. 8; Banco Mundial, 1994, p. i; Edwards y Foley, 1998, p. 38; Fowler, 2000, p. vii). Se espera que el enfoque basado en la sociedad civil pueda prevalecer ante los modelos de desarrollo que se centraron exclusivamente en el Estado o en el mercado (Pieterse, 1996; Hulme y Edwards, 1997, pp. 4 y 5; Brett, 1996, pp. 5 y 6). Precisamente, las organizaciones de la sociedad civil se han convertido en herramientas importantes para hacer más eficaces las políticas de reducción de la pobreza y para promover el cambio social, gracias a que su enfoque participativo de acción colectiva podría empoderar a los grupos pobres y vulnerables.

El reconocimiento del papel significativo de la sociedad civil ha tenido algunos efectos secundarios importantes. Las organizaciones de la sociedad civil se han convertido en significativos solicitantes de financiamiento para el desarrollo. El entusiasmo de los donantes por dar prioridad a la sociedad civil ha creado oportunidades para que las organizaciones

\footnotetext{
${ }^{1}$ Véase, por ejemplo, la página web del Banco Mundial http://www. worldbank.org/poverty/scapital .
} 
puedan establecerse, crecer, profesionalizarse y extender su área de influencia. Sin embargo, la gran cantidad de organizaciones y afiliaciones asociativas existente podría obedecer más a decisiones estratégicas, oportunidades de financiamiento y espíritu empresarial de asociación, que al auge de ánimo cooperativo en una población. Esta idea es apoyada por los investigadores que analizan los niveles extremadamente bajos de confianza y el predominio del clientelismo en los países en desarrollo. Algunas investigaciones científicas muestran que, en promedio, solamente el $16 \%$ de los latinoamericanos confía en su prójimo, mientras que en Europa esa proporción es de 60\% (Latinobarómetro, 2005, p. 32). La institución Transparencia Internacional afirma que países como Nicaragua han entrado en una situación de hipercorrupción, únicamente igualada por una cultura de tolerancia frente al incumplimiento y la defección. ${ }^{2}$ A nivel regional, la herencia compartida de desconfianza se refleja en los patrones sociales de interacción. La región latinoamericana parece estar caracterizada y dominada por el verticalismo y el clientelismo, los que básicamente revelan fragmentación e incapacidad para desarrollar una cooperación horizontal (Gambetta, 1988; Latinobarómetro, 2006). Estos elementos parecen indicar que, si hablamos de capital social en situaciones de bajo nivel de desarrollo socioeconómico, existiría una posible separación o incluso una contradicción entre las actitudes (confianza y cooperación horizontal) y las estructuras (vitalidad asociativa). A nivel político, esto podría significar que el enfoque basado en la sociedad civil no estaría necesariamente relacionado con la construcción, generación e implementación de confianza, cooperación horizontal y participación.

En consecuencia, el propósito principal de este artículo es investigar si en un escenario del Tercer Mundo se puede encontrar una relación entre la afiliación asociativa y los componentes 'actitudinales' del capital social, como en parte lo sugiere la literatura especializada.

Para aproximarse a este tema, se tratará de responder a las siguientes preguntas: ¿Está la vida asociativa ligada a las actitudes de confianza y cooperación horizontales,

${ }^{2}$ Véase http://www.ibw.com.ni/ ien/c5-7.htm y existe esta cooperación horizontal? ¿O está ligada, sobre todo, al acceso vertical y clientelista? En otras palabras: ¿qué es lo que promueven las asociaciones? ¿Desempeñan ellas con eficacia el papel de 'transformadoras', o solo despotrican contra las redes verticales y clientelistas? Como portadoras del cambio social, se espera que trabajen contra la lógica de tales redes, que mantienen a las personas pobres y a las vulnerables en posición de dependencia. En una situación de desarrollo ideal, la ayuda canalizada a través de las organizaciones debe fomentar y estimular patrones de interacción social cooperativos, confiables, inclusivos y horizontales. A esto cabe añadir la especial atención que se debe prestar al pobre y al vulnerable, dada la necesidad de modificar su posición de exclusión. Por lo tanto, resulta fundamental analizar si las organizaciones están llegando eficazmente a los grupos pobres.

Los datos que se presentan aquí fueron recogidos en virtud de una investigación propia llevada a cabo en 1999 en dos localidades campesinas nicaragüenses: el pueblo de El Toro, que cuenta con 103 familias, y el de La Danta, con 74 familias. En cada uno de ellos se entrevistó a 65 familias. En primer lugar, se examinaron las organizaciones, las estructuras de afiliación y los mecanismos de reclutamiento empleados. En segundo lugar, se recogieron datos sobre redes informales de cooperación horizontal. La información recogida muestra que las organizaciones dependen fuertemente de los líderes locales y de sus redes informales, lo cual indica que probablemente ellas no están cambiando los patrones sociales de interacción en los dos pueblos considerados, sino que los están reafirmando. Asimismo, su eficacia para llegar a los pobres también está altamente determinada por las redes locales. Los datos señalan que, si tales redes ya incluyen a los grupos pobres, las organizaciones llegan a ellos; de otro modo, el grupo vulnerable se mantiene fuera del alcance de estas y de sus intervenciones a favor del desarrollo.

Es importante mencionar que los datos recogidos no permiten analizar la dirección de la relación de causalidad, por lo que las ambiciones de este trabajo son modestas. El debate sobre la causalidad procedería si se obtuviera una relación significativa entre las organizaciones, la confianza y la reciprocidad. Pero sin una relación significativa entre esos fenómenos hay poco que discutir sobre la causalidad. 


\section{II}

\section{El panorama asociativo en los dos pueblos considerados}

El Toro y La Danta son pueblos campesinos aislados, situados en la zona más pobre de la región nicaragüense de Chinandega, muy cerca de la frontera con Honduras. Ambos son particularmente distintos. El Toro es uno de los pueblos de la reforma agraria, como se les conoce en Nicaragua: estos pueblos fueron organizados inicialmente en cooperativas de producción y obtuvieron grandes beneficios durante el régimen sandinista, en el decenio de 1980. Después de la derrota electoral de los sandinistas, en 1990, los activos de las cooperativas (tierra y ganado) fueron privatizados y divididos de manera equitativa entre los habitantes del pueblo respectivo. Además, sus poblaciones fueron ayudadas con frecuencia tanto por organizaciones de desarrollo nacionales e internacionales como por organizaciones no gubernamentales (ONG). Por lo tanto, El Toro era una comarca con mucha igualdad socioeconómica ya al inicio de la década de 1990, y su infraestructura está mucho más adelantada que la de La Danta. En El Toro hay pozos públicos de agua, electricidad, y más y mejor infraestructura para la educación y la salud; las casas son de concreto. La Danta, en cambio, no fue una comarca beneficiaria de la reforma agraria. Además, tanto la atención del gobierno sandinista como la de las ONG fueron, si no ausentes, muy limitadas. ${ }^{3}$ En consecuencia, no cuenta con muchos servicios básicos, carece de electricidad y pozos públicos, las casas son hechas de tierra y la infraestructura escolar y el puesto de salud se encuentran en un estado problemático.

Sin embargo, ambos pueblos tienen mucho en común. En El Toro y en La Danta más del $75 \%$ de la población depende de la agricultura y, en el momento

\footnotetext{
${ }^{3}$ Las entrevistas con encargados de proyectos, funcionarios de gobierno y coordinadores de las ong sugieren que, en general, los pueblos de la reforma agraria parecen tener más dificultades para adoptar conductas cooperativas y de confianza que los pueblos tradicionales. Los entrevistados indican que la fuerte presencia del partido sandinista y de las organizaciones nacionales e internacionales de solidaridad en dichos pueblos han consolidado una "cultura de falta de reciprocidad". La incesante corriente de beneficios hacia ellos sin esperar nada a cambio, señalan los entrevistados, es en parte responsable de la crisis de vínculos locales de reciprocidad y de cooperación. Por supuesto que estas cuestiones, que son solo parte de la situación, necesitan ser investigadas y no tan solo asumidas.
}

de la investigación, cerca del $40 \%$ de los agricultores de ambas comarcas carecía de tierras, lo cual significa que hay más o menos la misma cantidad de pobres en ambos pueblos. Importante es mencionar la evolución socioeconómica de El Toro, que pasó de una situación bastante igualitaria (gracias a la reforma agraria) a una condición de más desigualdad. ${ }^{4}$ En lo que respecta a la clasificación socioeconómica, se catalogó en el grupo más pobre a los que no tenían tierras, en el grupo medio a los agricultores dueños de menos de 50 hectáreas y en el grupo de elite a los agricultores que poseían 50 hectáreas o más. ${ }^{5}$ En cuanto a la preferencia política de los participantes, estos fueron catalogados como sandinistas (oposición de izquierda), liberales (partido de derecha en el poder) o sin preferencia. Respecto a la estructura asociativa, en ambos pueblos existe una cantidad similar de organizaciones con características parecidas. En cada uno hay tres grupos de intereses particulares (sindicatos, organizaciones de campesinos), nueve organizaciones no gubernamentales, cuatro instituciones gubernamentales y un comité comunal (cuadro 1).

Las organizaciones e instituciones presentes en los dos pueblos estudiados tienen en común un enfoque participativo del desarrollo. Asimismo, todas las organizaciones poseen vínculos importantes con el mundo exterior, pues se encuentran relacionadas con las autoridades municipales o con el gobierno central, sindicatos nacionales, oficinas de desarrollo regional y ONG nacionales e internacionales. La existencia de estos vínculos es fundamental para

\footnotetext{
${ }^{4}$ Esto significa que, en una década, El Toro ha pasado de una estructura más o menos igualitaria en cuanto a la tenencia de tierras, a una situación socioeconómica bastante desigual. Por falta de espacio, este artículo no examinará en detalle los mecanismos relacionados con esta evolución. Sin embargo, vale la pena mencionar que la falta de confianza ayudó a estimularla. Por esa falta de confianza los 'favores' y servicios se retribuyen en efectivo. Así, los habitantes tienen que pagar el acceso a ciertos servicios (a tierra, a bueyes, a información) y especialmente los pobres se endeudan cada vez más, por lo cual a largo plazo la venta de ganado, tierras y otros bienes se impone cuando las deudas se acumulan. De esta manera, algunos campesinos han ido cayendo gradualmente en más pobreza. Al respecto vale la pena recalcar que la reforma agraria, el partido sandinista y la solidaridad internacional contribuyeron a crear una cultura de no reciprocidad durante el decenio de 1980.

${ }^{5}$ Clasificación recogida del Ministerio de Agricultura de Nicaragua.
} 
Nicaragua (dos pueblos): Estructura de las organizaciones y afiliación a ellas

\begin{tabular}{|c|c|c|}
\hline Organizaciones & El Toro & La Danta \\
\hline Grupos de intereses & 2 & 3 \\
\hline Organizaciones no gubernamentales (ong) & 7 & 6 \\
\hline Instituciones gubernamentales que promueven el desarrollo de proyectos & 3 & 2 \\
\hline Comité comunal & 1 & 1 \\
\hline Número total de organizaciones & 13 & 12 \\
\hline \multicolumn{3}{|l|}{ Afiliación } \\
\hline Familias del pueblo con al menos una afiliación(\%) & 44 & 60 \\
\hline Familias del pueblo con más de una afiliación(\%) & 25 & 32 \\
\hline Promedio de afiliaciones por familia & 0,8 & 1,2 \\
\hline $\begin{array}{l}\text { Líderes locales que participan } \\
\text { (cuatro líderes en cada pueblo) }\end{array}$ & Todos & Todos \\
\hline \multicolumn{3}{|l|}{$\begin{array}{l}\text { Afiliación según la preferencia política } \\
\text { y el estatus socioeconómico de las familias del pueblo }\end{array}$} \\
\hline \multicolumn{3}{|l|}{ Familias del pueblo afiliadas, según su preferencia política (\%) } \\
\hline Entre las familias sandinistas & 79 & 93 \\
\hline Entre las familias liberales & 31 & 36 \\
\hline Entre aquellas sin preferencia política & 27 & 58 \\
\hline \multicolumn{3}{|l|}{ Familias afiliadas, según su estatus socioeconómico (\%) } \\
\hline Grupo de elite ( 50 hectáreas de tierras o más) & 57 & 80 \\
\hline Campesinos propietarios de tierras (menos de 50 hectáreas) & 84 & 68 \\
\hline Campesinos sin tierras & 20 & 64 \\
\hline \multicolumn{3}{|l|}{$\begin{array}{l}\text { Promedio de afiliaciones } \\
\text { según la preferencia política }\end{array}$} \\
\hline En el total de familias del pueblo & 0.8 & 1.2 \\
\hline En las familias sandinistas & 1.7 & 2.2 \\
\hline En las familias liberales & 0.3 & 0.5 \\
\hline
\end{tabular}

Fuente: elaboración propia.

todos los agentes implicados, pues les permite canalizar recursos tangibles e intangibles. Las semejanzas entre estas organizaciones (enfoque participativo, discurso sobre desarrollo y cooperación horizontal) contrastan fuertemente con la división teórica entre Estado y sociedad civil. Los agentes gubernamentales y no gubernamentales se desenvuelven en el mismo terreno, organizan la misma clase de intervenciones y utilizan enfoques similares en ambos pueblos. Las similitudes entre las organizaciones se refuerzan, sin duda, por el hecho de que todas ellas dependen de los donantes y se rigen por los lineamientos que ellos han establecido.

Para tener una visión más clara de las características de los miembros de estas organizaciones en ambos pueblos, las familias fueron segmentadas según sus preferencias políticas y su nivel socioeconómico, ${ }^{6}$ como lo resumió el cuadro 1.

${ }^{6}$ Cuando un agricultor tiene que preparar una hectárea de tierra para la siembra, solamente la escarda podría tomarle hasta 16 días de trabajo si lo hiciera sin ayuda. Si contratara a un trabajador, el tiempo
El gran número de organizaciones y los altísimos costos de afiliación (incluso múltiple) confirman que los habitantes de ambos pueblos están bastante organizados. Aun más, todos los líderes locales están involucrados en las actividades asociativas. Los cuatro líderes locales de uno y otro pueblo son sandinistas y se desempeñan como coordinadores de las organizaciones presentes en sus pueblos respectivos. Cada líder coordina por lo menos tres organizaciones.

Los participantes en organizaciones en ambos pueblos parecen disfrutar en su mayoría de una mejor posición económica relativa, dado que pertenecen a la elite o son campesinos dueños de tierras. Esto concuerda con el patrón encontrado en investigaciones anteriores: en general, la actividad asociativa tiende a estar relacionada con quienes no son extremadamente pobres.

de trabajo podría reducirse a la mitad (ocho días), pero tendría que pagarle a este aproximadamente 1,25 dólares por día, es decir, 10 dólares por ocho días de trabajo. 
Otros estudios también indican que la afiliación, la cooperación y la confianza parecen ser especialmente difíciles para los más pobres (Inglehart, 1988; Newton, 1997). No obstante, desde la perspectiva del desarrollo, las organizaciones deberían estar llegando precisamente a los grupos pobres y vulnerables. En La Danta, efectivamente, el grupo más pobre está considerablemente incluido en las asociaciones; sin embargo, en El Toro solamente el $20 \%$ de ese grupo participa.

En los dos pueblos examinados, los participantes parecen compartir una preferencia política por los sandinistas, característica que subsiste cuando se analiza la afiliación múltiple. En ambos se encontró habitantes que tienen hasta cinco afiliaciones, la mayoría de ellas sandinistas. El cuadro 1 indica claramente que en los dos pueblos el promedio de afiliaciones es considerablemente mayor entre los habitantes sandinistas, mientras que disminuye entre quienes optan por el partido liberal. En ambos pueblos llama la atención esta menor presencia de los liberales, pero en El Toro el sesgo relacionado con el nivel socioeconómico es aún más impresionante. Vemos así que en los dos pueblos la participación muestra un sesgo estructural. En todo caso, para comprender la estructura de la no participación tendremos que examinar los mecanismos asociativos de reclutamiento .

\section{III}

\section{Explicación de las preferencias}

Las preferencias por la afiliación sandinista están fuertemente relacionadas con la historia de un gran número de organizaciones en Nicaragua. En la década de 1980 el gobierno sandinista promovió y acogió todas las iniciativas asociativas de izquierda. Sin embargo, en la década de 1990 el auge del número de asociaciones, sobre todo progresistas, fue aún mayor. ${ }^{7}$ Aunque la mayoría de las organizaciones nacionales de izquierda se distanció del partido sandinista después de su derrota electoral en 1989, lo más probable es que esto no haya causado una diferencia apreciable en las estructuras de afiliación a nivel local. En todo caso, tomando en cuenta la historia del país, la mayor representación sandinista en las estructuras de afiliación era esperable.

Otra característica de suma importancia que se suele pasar por alto es que las organizaciones en países en desarrollo siempre significan proyectos y, por consiguiente, inyectan recursos a las comunidades. Los miembros parecen ser los primeros beneficiarios de estos recursos

\footnotetext{
${ }^{7}$ Obviamente, las ideas de excesiva confianza y control social coinciden con el debate respecto a crear lazos afectivos y tender puentes hacia el capital social. Demasiada confianza, especialmente sobre la base de un grupo de pertenencia, puede tener bastantes efectos negativos (véanse entre otros Woolcock, 1998; Portes, 1998; Granovetter, 1982). Sin embargo, los autores parecen coincidir en que siempre que se haga referencia al desarrollo colectivo, se debe preferir la existencia de redes de integración a la fragmentación y el aislamiento (Woolcock, 1998). Este artículo, por lo tanto, se centra principalmente en aquellos aspectos del capital social relacionados con la integración, la cooperación horizontal y la confianza, vistos como condiciones necesarias, pero no suficientes para el desarrollo democrático
}

tangibles e intangibles. En los pueblos, algunas organizaciones utilizan el sistema de 'alimento por trabajo'; por ejemplo, entregan alimentos a los agricultores que ponen en práctica con eficacia (nuevos) métodos y tecnologías agrícolas., y también les ofrecen materiales, como redes de alambre, zinc, bombas para rociar insecticidas y machetes. Algunas han proporcionado a sus miembros materiales de producción y ayuda financiera, mientras que otras les han brindado valiosa información de mercado sobre los precios del sésamo (o ajonjolî), lo cual consolida la posición de los agricultores al momento de negociar con los compradores intermedios. Todas las organizaciones ofrecen educación a través de talleres y seminarios. El volumen de recursos escasos que ellas manejan es impresionante, de modo que los beneficios de asociarse son tangibles. Si se tiene en cuenta que en ambos pueblos algunos habitantes acumulan hasta cinco afiliaciones, los conceptos de afiliación múltiple y de exclusión adquieren sorprendentemente un peso económico importante. Desde el punto de vista del acceso a los recursos, la afiliación se convierte en un privilegio.

En el ingreso a las organizaciones influyen fuertemente las redes informales. Así, el acceso a los recursos se regula por medio de tales redes, a través de lazos directos e indirectos. Esto convierte a los recursos en 'recursos sociales', ya que se insertan en las redes sociales (Lin, 1982, p. 132).

¿Por qué no es miembro de una organización?
Porque ellos no me dejan.
¿Quiénes son ellos? 


\section{Los líderes}

¿Qué quiere decir con eso?

Cuando una organización quiere hacer algo aquí, como un proyecto, siempre se arreglan con los líderes locales, y dicen: 'si conoces a algunas buenas personas, gente trabajadora que desee cooperar, reúnelas y comenzaremos con el proyecto'. $Y$ entonces, los líderes deciden quién va a estar en el grupo y escogen siempre a los mismos.

¿Le gustaría unirse?

Por supuesto... ellos están obteniendo beneficios y nosotros nunca conseguimos nada...”. (Agricultor de La Danta)

La cita anterior y la siguiente fueron recogidas en entrevistas efectuadas por la autora. La primera refleja la visión más común en estos pueblos. Quienes no participan se sienten excluidos de la vida asociativa, es decir, excluidos del acceso a los recursos. La afiliación es la estrategia que garantiza ese acceso. Por esta razón, las organizaciones pueden provocar conflictos entre los pobladores, debido al deseo de los líderes locales de ingresar a las redes con el fin de acceder a los recursos. Las entrevistas revelaron tensiones en los pueblos, porque ciertos habitantes son sistemáticamente incluidos mientras que otros permanecen fuera de las estructuras asociativas. Las organizaciones y los líderes locales confirmaron el importante rol de estos últimos cuando se trata de reclutar a los miembros:

"Sí, yo me encargo de seleccionar a las personas que van a trabajar en el proyecto. Algunas veces las organizaciones piden requisitos, como la edad o el sexo o la cantidad de tierra que deben poseer. Por supuesto que yo sigo sus instrucciones y escojo a la gente que conozco mejor, gente en la que puedo confiar. No puedo asumir la responsabilidad por gente a la que no conozco, porque si ellos 'meten la pata', entonces la organización me culpará a mí y perderé la relación".

(Líder local de La Danta)

Según Coleman (1990, p. 182), cabría argumentar que los líderes pueden ocupar la posición de intermediarios emprendedores. En estos agentes confían varios fideicomitentes (las organizaciones) para desplegar correctamente los recursos entre las autoridades (los miembros), quienes luego se ocupan de trabajar colectivamente para obtener los beneficios de la actividad (el proyecto). La responsabilidad de los líderes locales de seleccionar a los miembros simultáneamente descentraliza la ejecución del proyecto y la administración hacia los líderes locales. No obstante, dicha orientación parece ofrecer ventajas a las organizaciones, ya que los costos de transacción se reducen al apoyarse en redes sociales de confianza ya existentes. Esta tendencia a utilizar el capital social para garantizar la ejecución del proyecto puede ser cuestionada, especialmente cuando se utilizan las redes existentes sin poner en tela de juicio su composición, estructura y contenido, puesto que dichas redes pueden ser verticales, es decir, tratarse de redes clientelistas dependientes. En esencia, este uso instrumental de la confianza en el análisis y la práctica del desarrollo apunta en la misma dirección que las ideas de la escuela pragmática del capital social, la que concibe este capital como un instrumento para movilizar recursos.

En consecuencia, las redes son utilizadas para aumentar el control efectivo sobre la ejecución del proyecto a través de un grupo razonable (algunos líderes locales); estos líderes pueden ser considerados responsables y ser sancionados mediante el retiro de los proyectos (y, por ende, de los recursos) de la comunidad. Para evitar que esto suceda, el líder desplegará todos sus recursos de poder con miras a garantizar la realización del proyecto. Esto quiere decir que a su poder como intermediario empresarial sumará muy probablemente su poder como portador de acceso a los recursos que él mismo posee (tierra, bueyes, cantidades pequeñas de dinero y alimento).

Mientras más recursos controle un individuo, más jerárquica será la posición y más jerárquico el acceso a la organización. Quien desee acceder a esos recursos personales y sociales estará obligado a ofrecer compensaciones y a reciprocar (Lin, 1995, pp. 687 y 688). Además, los que obtengan ese acceso quedarán en deuda con quien se los brindó. Esto limita el control horizontal, la responsabilidad, la empoderación y la participación genuina, que son precisamente las metas del enfoque basado en la sociedad civil. Las diferencias de poder entre las asociaciones y los líderes locales, y entre los líderes locales y los habitantes, son demasiado grandes. La participación llega a ser entonces, en el mejor de los casos, adecuada para los de arriba y sancionadora para los de abajo. Según la evidencia disponible, en estos casos hay más inclinación hacia el clientelismo que hacia una participación genuina. Esto indica que ser parte de la vida asociativa tiene más que ver con los mecanismos del clientelismo que con el efecto horizontal del espíritu cooperativo. Siendo así, no parece creíble que las asociaciones puedan ser consideradas como manifestación o fuente de componentes 'actitudinales' del capital social. 
Por otro lado, se ha dicho ya que el fuerte sesgo sandinista observado se explica por la evolución histórica de las oportunidades políticas que han moldeado el carácter de la vida asociativa, al crearse y apoyarse principalmente en estructuras de liderazgo sandinista a nivel local. La consecuencia es que los liberales no pueden obtener ventaja de las oportunidades asociativas. Sin embargo, estos mecanismos no explican por qué en La Danta los líderes locales seleccionan a los campesinos más pobres para participar en las asociaciones, mientras que en El Toro esto no sucede. La no selección de los campesinos más pobres podría verse como algo absolutamente racional, dado que con más frecuencia se espera encontrar incumplimiento, desconfianza y defección en las clases socioeconómicas más bajas (Inglehart, 1988, p. 1213; Newton, 1997, p. 181). Asimismo, los pobres tienden a esperar poco del prójimo y del mundo en general (Newton, 1997): ellos tienen más que perder y suelen hacer finos cálculos sobre sus posibles riesgos, dado que la recompensa es incierta y el riesgo de defección es real. Así, en materia de reclutamiento, los líderes de El Toro parecen responder a esta lógica, aunque ella vaya en detrimento de las metas de desarrollo del enfoque basado en la sociedad civil que promueven los donantes, puesto que los recursos no alcanzan a los grupos más pobres. En La Danta, en cambio, los recursos llegan a una gran parte de los estratos más pobres, aunque cabe preguntarse por qué los líderes locales se arriesgan a asociarse con este grupo aparentemente poco confiable. La estructura y el contenido de las redes informales locales pueden dar respuesta a este interrogante.

\section{IV}

\section{Las redes cooperativas informales}

Según Coleman (1990), el surgimiento y la aplicación de normas se facilitan por el cierre (closure) de la red. Este "cierre" (closure) fomenta la honradez de las estructuras sociales y permite así la proliferación de obligaciones y expectativas. De otro modo podría ser difícil imponer sanciones. Las redes abiertas, por su parte, carecen de estos mecanismos de aplicación.

Regresando al estudio de las redes informales en los dos pueblos estudiados, se averiguará si es posible encontrar formas de cooperación horizontal en estas sociedades generalmente verticales y se analizará de qué manera esas formas se relacionan con el aspecto más clientelista de la vida asociativa. Se ha querido investigar aquí las formas de cooperación que se acercan a la dimensión horizontal y voluntaria del capital social. En los dos pueblos considerados, tal cooperación horizontal tomó la forma de intercambio de mano de obra entre agricultores vecinos. A esta relación cooperativa se la conoce como 'cambio de mano'. En su forma más simple, este mecanismo de intercambio informal rural se traduce en que el agricultor A ayuda al agricultor B algunos días en el campo de este último, y después el agricultor $\mathrm{B}$ devuelve el favor trabajando algunos días en el campo de A.

El mecanismo descrito es una considerable fuente de ahorro en un entorno en que el dinero es extremadamente escaso y la mano de obra muy costosa. Este tipo de redes cooperativas, por lo tanto, tiene gran importancia para los campesinos más pobres, puesto que les ahorra mucho dinero. Así, participar en estas formas de cooperación es un objetivo racional, a menos que haya falta de confianza. La confianza es fundamental en estas acciones, porque siempre hay lapsos de tiempo entre el favor hecho y el favor devuelto, de modo que el riesgo de defección es verdadero. Los agricultores entrevistados indicaron explícitamente que la confianza desempeña un papel fundamental en este mecanismo, sobre todo cuando se dan formas más complejas de 'cambio de mano' (con más de dos actores).

El mecanismo de confianza en esta clase de 'cambio de mano’ esencialmente funciona así: la persona A, que entrega el servicio, confía en que el beneficiario B

i) reconozca que le debe algo a $\mathrm{A}, \mathrm{y}$

ii) ejecute uno o más servicios a cambio (comparables en tamaño y/o calidad con el o los servicios prestados por A).

Es importante que A no tenga que ejercer presión para recibir un servicio a cambio. Si A debe invertir tiempo y energía en insistir ante B para que este devuelva el favor, se podría tensar la relación. Este mecanismo es en sí muy delicado. Los participantes se ofrecen espontáneamente favores y servicios 'a cambio', pero sin sobrecargar a la contraparte con demandas u obligaciones. Si después de cierto tiempo las partes sienten que ninguna de ellas está intentando 'quererlo todo', las relaciones cooperativas posiblemente prosperen hacia mecanismos de ayuda 
complejos y múltiples, en los cuales todos los involucrados pueden sacar ventajas de las capacidades, el conocimiento y las relaciones de los demás miembros de la comunidad cooperativa.

Además, los participantes en relaciones de 'cambio de mano' hicieron presente que su relación de 'trabajo por trabajo' implica en principio un 'intercambio igual'. La simetría entre las acciones de los actores es fundamental en esta relación. Si una de las partes comenzara a incumplir las obligaciones inherentes a la relación, podrían verse resquebrajadas la confianza y la reciprocidad. No cumplir con las expectativas puede tener consecuencias directas. Por otro lado, el mecanismo de responsabilidad en esta clase de relación es informal y horizontal. Para mantener viva la relación, es necesario ceñirse a los mecanismos que hacen a su horizontalidad: mantener la igualdad de derechos y obligaciones.

El cuadro 2 muestra que en El Toro solamente el 35\% de las familias del pueblo está involucrado en relaciones de 'cambio de mano', mientras que en La Danta la proporción alcanza al 78\%. Esto indica que en La Danta las personas, en su gran mayoría, cooperan horizontalmente unas con otras. Una característica importante de las redes es la de estar o no conectadas. Los actores de una red desconectada pueden hallarse agrupados en dos o más subredes que no estén relacionadas unas con otras. Así, mientras más subredes tenga una red, más desconectada estará (Wasserman y Faust, 1994, p. 109). Podemos ver que en La Danta un grupo muy grande está organizado en solo cinco subredes. Por otra parte, en El Toro un grupo pequeño de agricultores está organizado en 10 subredes desconectadas. Las subredes, sin embargo, no dan información específica sobre la densidad de las redes. En una red densa, cada par de actores está conectado a través de una vía, de modo que todos los agentes son mutuamente accesibles. El coeficiente de accesibilidad es entonces igual a 1, su valor máximo. En La Danta dicho coeficiente es de 0.59 y en El Toro de 0.12. El análisis de las redes también mostró que, en promedio, el número de relaciones de 'cambio de mano' en La Danta es más alto que en El Toro. Esto significa que en La Danta no solo hay más familias implicadas en el 'cambio de mano', sino también que en promedio cada familia mantiene más relaciones de este tipo con otras familias. Además, en La Danta todos los líderes asociativos están involucrados en relaciones de 'cambio de mano', mientras que en El Toro ninguno de los líderes locales mantiene este tipo de relaciones de cooperación con los lugareños.

En general, la cooperación y la confianza horizontales, al parecer, son considerables en La Danta. La alta densidad indica un casi cierre (closure) de las redes y esto las hace más eficaces en términos de conformidad con las normas de reciprocidad. Por el número de familias implicadas y porque los líderes locales están sujetos a estas normas de responsabilidad, el pueblo de La Danta parece menos jerárquico, más inclusivo e integrado, y exhibe más confianza y reciprocidad horizontales que El Toro. Además, la presencia de los líderes locales en las redes cooperativas de $\mathrm{La}$ Danta sugiere que ellos están sujetos a mecanismos

CUADRO 2

\section{Nicaragua (dos pueblos): Estructura de las redes informales de cooperación} y características de sus miembros

\begin{tabular}{lcc}
\hline Estructura de la red & El Toro & La Danta \\
\hline Familias del pueblo involucradas en la red (\%) & 35 & 78 \\
Cantidad de subredes en el pueblo & 10 & 5 \\
Coeficiente de accesibilidad & 0,12 & 0,59 \\
Promedio de relaciones de 'cambio de mano' por familia & 0,6 & 1,8 \\
Líderes locales que participan & Ninguno & Todos \\
Características de las familias miembros & & \\
Participación de las familias del pueblo según su preferencia política (\%) & & 89 \\
$\quad$ Sandinistas & 55 & 80 \\
$\quad$ Liberales & 31 & 63 \\
Sin preferencia & 40 & 80 \\
Participación de las familias del pueblo según su estatus socioeconómico & \\
$\quad$ Elite (50 hectáreas de tierras o más) & 29 & 71 \\
Campesinos propietarios de tierras (menos de 50 ha) & 55 & 40 \\
Campesinos sin tierras & & \\
\hline
\end{tabular}

Fuente: elaboración propia. 
de control social interno. En El Toro, la ausencia de líderes asociativos en la red cooperativa informal podría indicar que estos escapan a los mecanismos de responsabilidad horizontal, al intercambio igualitario y a las expectativas y obligaciones mutuas. El control social de la conducta desviada, por lo tanto, sería mucho más fácil en La Danta que en El Toro.

Los actores involucrados en uno y otro pueblo parecen ser primordialmente sandinistas y propietarios de tierras. Estos datos coinciden con el perfil de los participantes expuesto con anterioridad. Sin embargo, existe una discrepancia un tanto curiosa. Mientras que en La Danta los liberales estuvieron fuertemente subrepresentados a nivel de afiliación, en esta red cooperativa se ha involucrado no menos del $80 \%$ del grupo liberal (cuadro 2). Del mismo modo, mientras que en El Toro los campesinos sin tierras están casi totalmente ausentes de la vida asociativa, en La Danta vemos que cerca del $40 \%$ de ellos coopera de manera informal en el 'cambio de mano'. Después de comparar los datos de la vida asociativa con los datos sobre las relaciones de cooperación, surge el patrón siguiente: en El Toro la mitad de las familias no involucradas en las estructuras formales de participación practica relaciones de 'cambio de mano', y lo mismo hace la mitad de las familias que sí están involucradas en esas estructuras formales. En La Danta, practican el 'cambio de mano' 20 de las 29

\section{$\mathrm{V}$}

\section{Conclusión}

La existencia de gran cantidad de asociaciones y de altos niveles de afiliación en ellas no está relacionada necesariamente con la confianza, la reciprocidad y la cooperación horizontal. Las asociaciones no siempre son la representación estructural de los componentes 'actitudinales' del capital social. Los dos pueblos estudiados presentan puntajes similares respecto a la vida asociativa, pero mientras en El Toro son solo unos pocos grupos pequeños los que cooperan de manera horizontal, en La Danta una gran cantidad de personas participa en las redes horizontales.

Se vio que en ambos pueblos la vida asociativa efectivamente está sujeta más bien a patrones de interacción clientelistas: los actores tratan de acceder a los recursos a través de líderes locales que desempeñan el papel de intermediarios entre las organizaciones y los habitantes. Pero al analizar las redes cooperativas informales surgió familias no involucradas en las estructuras formales y 34 de las 41 familias que sí están involucradas en ellas.

Entonces, puede decirse que la vida asociativa no está ligada necesariamente a los componentes 'actitudinales' del capital social. Se ha visto que los grupos que estaban subrepresentados en las estructuras formales de participación en la vida asociativa se encuentran muy presentes en las redes de cooperación horizontales. En ambos casos, la participación formal en actividades asociativas y procesos de desarrollo parece no guardar mucha relación con el espíritu cooperativo. Los mecanismos de reclutamiento y los datos sobre las estructuras de cooperación afiliativas muestran la discrepancia entre ambos fenómenos.

Más importante aún es comprobar que la fuerte integración de los pobres en las redes de La Danta reduce el riesgo de defección en las actividades de la vida asociativa. Dado que las redes proporcionan un entorno en que generalmente se espera reciprocidad, los líderes pueden incluir a los más pobres sin arriesgarse demasiado a grandes problemas de incumplimiento. La participación activa de numerosos grupos de pobres en redes horizontales (especialmente en La Danta y en menor grado en El Toro) parece cuestionar la visión tradicional de América Latina, según la cual los más pobres permanecen marginados, aislados, no organizados, algo fatalistas y dependientes de patrones ricos (Huntington y Nelson, 1976). una visión muy distinta: este enfoque permitió acercarse a los componentes 'actitudinales' del capital social, y se vio que los dos pueblos diferían bastante en cuanto a cooperación horizontal. Si se hubiera confiado en la aparente relación entre las asociaciones y el capital social, se hubiera concluido que ambos exhibían puntajes más o menos similares en materia de capital social.

Este descubrimiento de los mecanismos relacionados con las redes de 'cambio de mano' revela que quizás estas sean un indicador alternativo de la dimensión cooperativa horizontal y voluntaria del concepto de capital social. Es necesario encontrar esta clase de indicadores para deconstruir el complejo concepto de capital social y sus supuestos normativos (Edwards y Foley, 1998), especialmente en los países en desarrollo, puesto que las implicaciones políticas pueden ser significativas. 
Se señaló previamente que, en la cooperación para el desarrollo, el enfoque basado en la sociedad civil está cargado de grandes esperanzas. Los donantes esperan que las organizaciones puedan forjar un cambio social al trabajar contra el principio clientelista y vertical y estimular la inclusión de los grupos pobres y vulnerables. Las modalidades más recientes de cooperación para el desarrollo optan claramente por dar un lugar muy importante a las organizaciones de la sociedad civil en el proceso de reducción de la pobreza (Fowler, 2000; Tikare, Youssef y otros, 2002; Booth, 2003). Pero lo presentado aquí muestra que estos objetivos son algo simplistas, sobre todo, dadas las realidades complejas que enfrentan los países en desarrollo. Se ha visto que en el caso de Nicaragua el panorama asociativo no es tan pluralista ni tan inclusivo como se suele suponer. Por lo tanto, reforzar la sociedad civil para que esta forje un cambio social puede significar una contradicción, porque es posible que los mismos elementos que frenan el cambio social estén enraizados en el funcionamiento de la sociedad civil.

La historia y la evolución de las oportunidades políticas matizan la composición actual de la sociedad civil y la estructura de afiliación dentro de las localidades. Hemos visto que en los dos pueblos considerados ciertos grupos, en este caso los habitantes sandinistas, están mejor organizados que otros, obtienen más fácilmente el acceso a organizaciones y exhiben estructuras de liderazgo sostenidas por algunas que tienen su origen fuera de la comarca (en la ciudad, en la capital, en el extranjero). Las organizaciones descentralizan su funcionamiento mediante la transferencia de poder a los líderes locales, quienes entonces pueden escoger a los participantes y también distribuir los recursos. De esta manera, la eficacia de las intervenciones con miras al desarrollo que efectúan las organizaciones parece quedar determinada en gran parte por la estructura local de las redes informales que las nutren y refuerzan. Si estas redes informales son relativamente inclusivas, horizontales, densas y regidas por mecanismos de responsabilidad, y si en ellas se posicionan los líderes y los seguidores pobres y ricos (como en La Danta), contribuirán a lo que las asociaciones están apoyando, desarrollando y estimulando. Pero si las redes son excluyentes y clientelistas, las asociaciones con sus proyectos también estarán contribuyendo a este tipo de organización social. Desde tal punto de vista, la 'participación' puede reproducir y hacer que avance la dominación de líderes y elites (Kapoor, 2004, p. 2, y 2005, p. 1210).

La inserción de los líderes locales en las redes cooperativas informales evita que estos abusen de su posición de poder, pues los sujeta a mecanismos internos de control social a través de redes densas. Así, las redes en La Danta promueven la honradez (Coleman, 1988 y 1990), pero no sucede lo mismo en El Toro. Los líderes locales de este último pueblo no participan en las redes cooperativas horizontales, las redes mismas se encuentran bastante fragmentadas y es pequeña la cantidad de gente que participa en ellas; esto, según Coleman, revela la dificultad de crear un ambiente confiable donde se mantenga la conformidad a la norma y la reciprocidad. Así, pues, cuando las asociaciones tienen que relacionarse con entornos parecidos al de El Toro, efectivamente están institucionalizando el verticalismo y el clientelismo, y reforzando la posición de poder desenfrenado de los líderes. Por lo tanto, es obvio que en ambos pueblos las relaciones horizontales y de confianza se dan especialmente en las redes informales y mucho menos en las redes formales. Parece así muy poco probable que estas últimas puedan ser consideradas fuentes de confianza. En los dos pueblos hay grupos que cooperan horizontalmente y tienen relaciones de confianza, aunque no acceden a las organizaciones, ya sea por tener la preferencia política incorrecta en La Danta, o por no estar vinculados con los líderes locales en El Toro (porque estos últimos están ausentes de las redes horizontales). Lo malo es que las organizaciones también consolidan e institucionalizan esta exclusión. La estructura de participación sesgada probablemente guarda más relación con redes informales sesgadas, lo cual indica divisiones. Las divisiones en sí no son problemáticas mientras todos los grupos puedan acceder a los recursos escasos dentro de sus respectivas estructuras. Sin embargo, este no es el caso en los pueblos estudiados, porque el panorama asociativo no se encuentra suficientemente diversificado o porque las organizaciones carecen de las capacidades, el tiempo o los medios para profundizar el estudio de las localidades en las cuales podrían llegar a ser activas.

Estos alcances nos llevan a formular algunas recomendaciones en términos de política. Ante todo, el enfoque basado en la sociedad civil dentro de la práctica del desarrollo necesita urgentemente de una revisión crítica. Las connotaciones románticas y positivas que se ligan al papel, la función y los efectos de una sociedad civil grande y vibrante en materia de democracia y desarrollo económico, pueden resultar simplistas o incluso incorrectas en países en desarrollo. Apostar por los enfoques únicos que los donantes a menudo imponen niega la idea de la importancia de los contextos. Parece utópico entonces el deseo de crear capital social como si fuera un simple recurso, un resultado esperable de la inversión en un instrumento dado. 
Si se tiene en cuenta el contexto, surgen algunas preguntas y observaciones. Al estimular las organizaciones de la sociedad civil no se estimulan necesariamente aquellas actitudes que tendemos a relacionar con el capital social. Si la consolidación de la sociedad civil es vital, ¿parece también vital apoyar la diversificación de la sociedad civil, o se la debe aceptar como viene? ¿Es la participación una buena herramienta en cualquier contexto? Para llegar a los pobres en poblaciones fragmentadas, clientelistas y desconfiadas talvez sea preciso confrontar a ciertos líderes o hacerlos competir. Es probable que la solución a este problema tenga que venir de arriba hacia abajo, mediante la imposición de criterios de selección o inclusión transparentes. Solo así se podría trabajar realmente en beneficio de los grupos más débiles (Van der Linden, 1997; Vandana, 1996). En otras palabras, si la inclusión de ciertos grupos no surge automáticamente desde abajo, hay que establecerla y controlarla desde arriba; esto implica la imposición e implementación eficaz, de arriba hacia abajo, de mecanismos transparentes -incluidas las sanciones en caso de defección- a nivel de las organizaciones y de las intervenciones orientadas al desarrollo.

Esta es, irónicamente, la conclusión contradictoria de la presente investigación: la institucionalización de la desconfianza es la única vía para contrapesar el clientelismo y la exclusión arbitraria. La creación de confianza y, por ende, de capital social depende entonces del funcionamiento transparente de las instituciones, proyectos e intervenciones y de la posibilidad de evitar y sancionar el abuso clientelista de los recursos. Por lo tanto, las organizaciones donantes (gubernamentales y no gubernamentales) no deberían traspasar todo el poder a sus interlocutores locales (los líderes), sino mantener un control mucho más firme sobre el uso de los recursos y los mecanismos de inclusión y exclusión .

Todas estas observaciones apuntan a la necesidad de efectuar más investigaciones desde el campo del desarrollo. En primer lugar, es importante llevar a cabo estudios ex ante del lugar, para conocer la composición de una comunidad dada y establecer cuál es la relación entre sus diversos grupos. Parece indispensable evitar que se consoliden las posiciones monopolizadas por los líderes, diversificar las fuentes de información y acrecentar las oportunidades de alcanzar a los grupos realmente pobres y vulnerables. Descubrir hasta dónde llegan las redes de los líderes y cuáles son sus criterios de inclusión y acceso a ellas podría, señalar también dónde debería iniciarse el verdadero trabajo a favor del desarrollo.

\section{Bibliografía}

Banco Mundial (1994): The World Bank and Participation, Washington, D.C.

Booth, D. (2003): Introduction and overview, Development Policy Review, vol. 21, No 2, Oxford, Reino Unido, Blackwell Publishing.

Brett, E.A. (1996): The participatory principle in development projects: the costs and benefits of cooperation, Public Administration and Development, vol. 16, № 1, Nueva York, John Wiley \& Sons.

Coleman, J.S. (1988): Social capital in the creation of human capital, American Journal for Sociology, vol. 94, Chicago, The University of Chicago Press.

(1990): Foundations of Social Theory, Cambridge, Harvard University Press.

Edwards, B. y M.W. Foley (1998): Social capital and civil society beyond Putnam, American Behavioral Scientist, vol. 42, $\mathrm{N}^{\circ} 2$, Londres, Sage Publications, septiembre.

Fowler, A. (2000): Civil Society, NGDOs and Social Development: Changing the Rules of the Game, Occasional Paper, $\mathrm{N}^{\mathrm{o}} 1$, Ginebra, Instituto de Investigaciones de las Naciones Unidas para el Desarrollo Social.

Fukuyama F. (1995): Trust: The Social Virtues and the Creation of Prosperity, New York, Free Press.

Gambetta, D. (1988): Trust: Making and Breaking Cooperative Relations, Oxford, Blackwell.

Granovetter, M. (1982): The strength of weak ties: a network theory revisited, en P. Marsden y N. Lin, Social Structure and Network Analysis, Londres, Sage Publications.

Harrison, L.E. y S.P. Huntington (comps.) (2000): Culture Matters: How Values Shape Human Progress, Nueva York, Basic Books.
Hulme, D. y M. Edwards (comps.) (1997): NGOs, States and Donors, Too Close for Comfort?, Londres, MacMillan.

Hume, D. (1923): Tratado de la naturaleza, Madrid, Calpe.

Huntington, S.P. y J.M. Nelson (1976): Political Participation in Developing Countries: No Easy Choice, Londres, Harvard University Press.

Inglehart, R. (1988): The renaissance of political culture, American Political Science Review, vol. 82, No 4, Washington, D.C., American Political Science Association.

Kapoor, I. (2004): The power of participation, Current Issues in Comparative Education, vol. 6, No 2, Nueva York, Teachers College, Columbia University.

(2005): Participatory development, complicity and desire, Third World Quarterly, vol. 26, № 8, Londres, Routledge.

Latinobarómetro (2005): Informe Latinobarómetro 2005, Santiago de Chile. Disponible en www.latinobarometro.org.

(2006): Informe de prensa 2006. Disponible en www. latinobarometro.org

Lin, N. (1982): Social resources and instrumental action, en P. Marsden y N. Lin (comps.), Social Structure and Network Analysis, Londres, Sage Publications.

(1995): Social resources: a theory of social capital, Revue Française de Sociologie, vol. 36, № 4, París, Éditions OPHRYS/ Association Revue Française de Sociologie.

Newton, K. (1997): Social capital and democracy, American Behavioral Scientist, vol. 40, № 5, Londres, Sage Publications.

Ostrom, E. (2001): Social Capital and Collective Action, documento presentado en la conferencia "Social Capital: Interdisciplinary 
Perspectives" (Exeter, Exeter University, 15 al 20 de septiembre de 2001).

Pieterse, J.N. (1996): My Paradigm or Yours? Alternative Development, Post-Development, Reflexive Development, ISs Working Paper, $\mathrm{N}^{\circ} 229$, La Haya, Instituto de Estudios Sociales.

PNUD (Programa de las Naciones Unidas para el Desarrollo) (1993): Human Development Report 1993, Nueva York, Oxford University Press.

Portes, A. (1998): Social capital, its origins and applications in modern sociology, Annual Review of Sociology, vol. 24, Palo Alto, California, Annual Reviews.

Putnam, R.D. (1993): Making Democracy Work: Civic Traditions in Modern Italy, Princeton, Princeton University Press. (2000): Bowling Alone: the Collapse and Revival of American Community, Nueva York, Simon and Schuster.
Tikare, S., D. Youssef y otros (2002): Participation, PRSP Sourcebook, Washington, D.C., Banco Mundial.

Vandana, D. (1996): Access to power and participation, Third World Planning Review, vol. 18, № (2), Liverpool, Liverpool University Press.

Van der Linden, J. (1997): On popular participation in a culture of patronage: patrons and grassroots organizations in a sites and services project in Hyderabad, Pakistan, Environment and Urbanisation, vol. $9, \mathrm{~N}^{\circ} 1$, Londres, Instituto Internacional para el Medio Ambiente y el Desarrollo.

Wasserman, S. y K. Faust (1994): Social Network Analysis: Methods and Applications, Cambridge, Cambridge University Press.

Woolcock, M. (1998): Social capital and economic development: toward a theoretical synthesis and policy framework, Theory and Society, vol. 27, $\mathrm{N}^{\circ}$ 2, Nueva York, Springer, abril. 